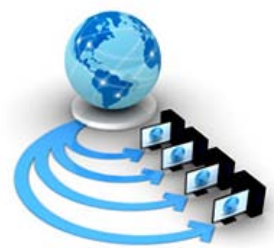

Volume 8, No. 7, July - August 2017

International Journal of Advanced Research in Computer Science

RESEARCH PAPER

Available Online at www.ijarcs.info

\title{
AUTOMATED HYBRID ALGORITHM FOR BLOOD CELL SEGMENTATION, RBC \& WBC DETECTION
}

\author{
Jay Shankar sharma \\ Computer Science and Engineering \\ Apex Institute of Engineering and technology \\ Jaipur, India
}

\author{
Brij Kishore \\ Computer Science and Engineering \\ Apex Institute of Engineering and technology \\ Jaipur, India
}

\begin{abstract}
Detection and diagnosis of a disease, at the appropriate early stage and time is crucial to save is in the field of medicine. The time in sever as emergency cased for non-automated tests such as blood tests can load a significant increase in mortality rates. As imaging techniques are widely used in the field of medical sciences for the detection of various diseases, classification of famous cancers, etc.

Also the existing system used by pathologists for the identification and classification of blood parameters is expensive and time-consuming. As the main power included in non-transformed festivals is high, they tend to be costly and are not affordable for an average patient.

Therefore, in this thesis we propose a novel image processing technique that can be automated by identifying the blood parameters such as red blood cell count, white blood cell count and other parameters with precision compared to other existing techniques. The white blood cell count, two algorithms we use each and its weighted average according to the user's performance is treated as final result.

Red blood cell count is achieved by Hough's circular transformation and segmented red blood cell count. Similarly, the white blood cell count is achieved using the k-media pool and the segmented blood count in the blue channel with the area response. The proposed technique also helps segregate blood cells into different categories

Keywords: Blood Cell Segmentation, RBC, WBC Count, Hough Transform, Blob Analysis, Image Processing, MATLAB.
\end{abstract}

\section{INTRODUCTION}

The blood consists of a suspension of special cells in a liquid called plasma. Blood consists of $55 \%$ plasma, and $45 \%$ by cells called formed elements. The blood performs a lot of important functions. By means of the hemoglobin contained in the erythrocytes, it carries oxygen to the tissues and collects the carbon dioxide (CO2). It also conveys nutritive substances (e.g. amino acids, sugars, Mineral salts). [1] The blood is circulating fluid, providing nutrition, oxygen and waste for the body. The blood is mainly liquid, which is suspended with many cells and proteins, making the blood "thicker" than pure water. Most people have about 5 liters (more than a gallon) of blood. Called liquid plasma accounted for half of the blood content. The plasma contains proteins that help the blood clot, transport substances through blood and perform other functions. The plasma also contains glucose and other dissolved nutrients. Red blood cells (RBCs), also called erythrocytes, are the most common type of blood cell and the vertebrate organism's principal means of delivering oxygen (O2) to the body tissues-via blood flow through the circulatory system. RBCs take up oxygen in the lungs or gills and release it into tissues while squeezing through the body's capillaries. [2]

The cytoplasm of erythrocytes is rich in hemoglobin, an iron-containing biomolecule that can bind oxygen and is responsible for the red color of the cells. The cell membrane is composed of proteins and lipids, and this structure provides properties essential for physiological cell function such as deformability and stability while traversing the circulatory system and specifically the capillary network.

White blood cells (WBCs) are also known as leukocytes. They can be divided into granulocytes and agranulocytes. The former have cytoplasms that contain organelles that appear as coloured granules through light microscopy, hence their name. Granulocytes consist of neutrophils, eosinophils and basophils.
In contrast, agranulocytes do not contain granules. They consist of lymphocytes and monocytes.

All white blood cells have nuclei, which distinguishes them from the other blood cells, the enucleated red blood cells (RBCs) and platelets. Types of white blood cells can be classified in standard ways. Two pairs of broadest categories classify them either by structure (granulocytes or granulocytes) or by cell division lineage (myeloid cells or lymphoid cells).

Platelets are small fragments of bone marrow cells and are therefore not really classified as cells themselves. Platelets have the following functions:

1. Secrete vasoconstrictors which constrict blood vessels, causing vascular spasms in broken blood vessels

2. Form temporary platelet plugs to stop bleeding

3. Secrete procoagulants (clotting factors) to promote blood clotting

4. Dissolve blood clots when they are no longer needed

5. Digest and destroy bacteria

6. Secrete chemicals that attract neutrophils and monocytes to sites of inflammation

Secrete growth factors to maintain the linings of blood vessels

Blood has several roles in inflammation:

- Leukocytes, or white blood cells, destroy invading microorganisms and cancer cells

- Antibodies and other proteins destroy pathogenic substances

Platelet factors initiate blood clotting and help minimize blood loss. [3, 4, 5]

\section{LITURATURE REVIEW}

The eosinophil cells are one of the many variable constituents that flow in the plasma of the blood. By using various techniques available in digital image processing these cells can be identified and counted. Through this paper, a 
method is proposed that will identify eosinophil cells in a much cheaper and less time consuming manner using the Hue Saturation Intensity ( HSI) color model, and the 8-Connected component labelling algorithm to count the number of identified eosinophil cells in a given blood smear image. Input images were collected from a pathological lab by placing a camera on the microscopes eyepiece. About twenty images were captured from the blood smears prepared by the pathologists of twenty different patients. The original image in the RGB color model was then converted to the HSI color model. From here the work was focused on the image representing the hue values. This image representing the hue values was converted into grayscale to make the identification of the eosinophils efficient. The red, green and blue pixel value of the eosinophil cell in the grayscale image was found to be zero. The image is then converted into a binary image to make to apply the 8-connected component labeling algorithm to count and label the eosinophil cells. The accuracy of the proposed system calculated was $85 \%$. [6]

Blood cell detection and counting is the initial process for detecting and diagnosing diseases. Several image processing algorithms are there for the blood cell classification and counting. The processed image helps to detect different blood related diseases. In all those algorithms several pre-processing steps are there for the process of detection and counting. Though all the algorithms give accurate results, the preprocessing steps are complex and time-consuming. This paper discusses about the RBC and WBC detection using fuzzy logic. Fuzzy logic toolbox software in MATLAB is used to develop the model on virtual platform. The goal of this research work is to produce cost effective and efficient computer vision system for automatic counting of blood cells from the blood smear microscopic image. In this paper we have detected RBCs and WBCs using fuzzy logic and the count is displayed. This algorithm provides accurate count and the time taken to complete the process is also very less. [7]

The measure of WBC and RBC Cells are very crucial to diagnose various diseases. Diseases like anemia, leukemia etc. can easily diagnose by calculation of WBC and RBC. Healthcare industries are focusing on the approach to generate report of blood cell count in fast and cost-effective way. Conventional method of manual measurement of red blood cell under a microscope yields incorrect results, consumes more time and very expensive. In market, there are numerous systems available for the automatic quantification of blood cells. These systems allow counting the number of different types of cells within the blood smear slides. The aim objective of this research is to produce a survey on computer vision system used image processing algorithms to detect and estimate the number of red blood cells in the blood sample image. In this project, image processing algorithms are used for counting of blood cells. [8] Image processing algorithms involve six major steps: image acquisition, pre-processing, image enhancement, image segmentation, feature extraction and counting algorithm. In this project, segmentation, detection, and counting red blood cells in the blood sample image is carried out using Hough Transform, Roughest theory and KNN method. Image processing techniques are helpful for object counting and reduce the time of counting effectively. Proper recognition of the object is important for object counting. The accuracy of the algorithm depends on camera used, size of objects, whether or not objects touching and illumination conditions. In this project, presents software based solution for counting the blood cells and detect blood disease type. Proposed method of cell counting is fast, cost effective and produces accurate results. It can be easily implemented in medical facilities anywhere with minimal investment in infrastructure. This method can also recognize the overlapping cells and counts them separately. [9]

\section{Methodology}

In computer vision and image processing, Otsu's method, named after Nobuyuki Otsu , is used to automatically perform clustering-based image thresholding, or, the reduction of a gray level image to a binary image. The algorithm assumes that the image contains two classes of pixels following bi-modal histogram (foreground pixels and background pixels), it then calculates the optimum threshold separating the two classes so that their combined spread (intra-class variance) is minimal, or equivalently (because the sum of pairwise squared distances is constant), so that their inter-class variance is maximal. Consequently, Otsu's method is roughly a one-dimensional, discrete analog of Fisher's Discriminant Analysis. [10]

This method usually increases the global contrast of many images, especially when the usable data of the image is represented by close contrast values. Through this adjustment, the intensities can be better distributed on the histogram. This allows for areas of lower local contrast to gain a higher contrast. Histogram equalization accomplishes this by effectively spreading out the most frequent intensity values. The method is useful in images with backgrounds and foregrounds that are both bright or both dark. In particular, the method can lead to better views of bone structure in $\mathrm{x}$-ray images, and to better detail in photographs that are over or under-exposed. A key advantage of the method is that it is a fairly straightforward technique and an invertible operator. [11]

Enhancements are used to make it easier for visual interpretation and understanding of imagery. The advantage of digital imagery is that it allows us to manipulate the digital pixel values in an image. Although radiometric corrections for illumination, atmospheric influences, and sensor characteristics may be done prior to distribution of data to the user, the image may still not be optimized for visual interpretation. Remote sensing devices, particularly those operated from satellite platforms, must be designed to cope with levels of target/background energy which are typical of all conditions likely to be encountered in routine use. With large variations in spectral response from a diverse range of targets (e.g. forest, deserts, snowfields, water, etc.) no generic radiometric correction could optimally account for and display the optimum brightness range and contrast for all targets. Thus, for each application and each image, a custom adjustment of the range and distribution of brightness values is usually necessary.

The Hough transform in its simplest form is a method to detect straight lines but it can also be used to detect circles or ellipses. The algorithm assumes that the edge is detected and it is robust against noise or missing points.

The parameterization of Circular hough transform: A circle can be described completely with three pieces of information: the center $(\mathrm{a}, \mathrm{b})$ and the radius. (The center consists of two parts, hence a total of three)

$$
\begin{aligned}
& x=a+R \cos \theta \\
& y=b+R \sin \theta
\end{aligned}
$$

When the $\theta$ varies from 0 to 360 , a complete circle of radius $\mathrm{R}$ is generated.

So with the Circle Hough Transform, we expect to find triplets of $(\mathrm{x}, \mathrm{y}, \mathrm{R})$ that are highly probably circles in the image. 
That is, we want to find three parameters. Thus, the parameter space is 3D... meaning things can get ugly if you don't tread slowly. Out of memory errors are common even if your programming language uses virtual memory. [12, 13, 14]

K-means is one of the simplest unsupervised learning algorithms that solve the well known clustering problem. The procedure follows a simple and easy way to classify a given data set through a certain number of clusters (assume $\mathrm{k}$ clusters) fixed a priori. The main idea is to define $\mathrm{k}$ centroids, one for each cluster. These centroids should be placed in a cunning way because of different location causes different result. So, the better choice is to place them as much as possible far away from each other. The next step is to take each point belonging to a given data set and associate it to the nearest centroid. When no point is pending, the first step is completed and an early groupage is done. [15]

At this point we need to re-calculate $\mathrm{k}$ new centroids as barycenter of the clusters resulting from the previous step. After we have these k new centroids, a new binding has to be done between the same data set points and the nearest new centroid. A loop has been generated. As a result of this loop we may notice that the $\mathrm{k}$ centroids change their location step by step until no more changes are done. In other words centroids do not move any more.

First we take Read Image of stained Blood Cell Slide than Convert image to Gray scale after that apply linear contrast stretching on to Gray scale image using Im adjust Function than we apply Histogram equalization Technique after that Obtain background brightening by combining Im adjust \& Histogram equalize images using image addition than highlight component using subtraction of background brightened \& Histogram equalized images using image subtraction after that we remove components of Non-interest by addition of background brightened image \& highlighted component image using image addition than we apply 2-D order statistic filtering technique using $1^{\text {st }}$ order $\&$ domain a unity matrix of size $3 \times 3$ after that,

First We Take Start And Read Image of stained Blood cell slide than Extract Red Dimension of the image using matrix operation than Perform adaptive Histogram Equalization of Red Dimension image after that apply circular hough transform of fogram equalize image Using parameters of Radius size From 5 to 25 pixels Gradient magnitude threshold 20, local maximal filter radius 13 \& multi red to 1 (lowest tolerance). [16, 17]

Frist we Start than WE Take Read Image of Stained Blood Cell Slide after that Convert Colour Space From RGB to HSV than Apply 2-D Medion filter on Each Component of HSV Colour Space Image i.e. Hue saturation \& value. Filter Neighbor Hood Selected is $5 \mathrm{X} 5$ after that apply 2-D Medion filter on each Component of RGS colour Space i.e. Red, Green \& Blue Components Filter Neighbor Hood Selected is 5X5 than Extract Green Channel From RGB Image Using Matrix Operation in Ig1 after that Compute Gray Threshold of Green Channel Image Using Ostu's Method than Obtain binary image Using $\mathrm{E}=(\operatorname{Ig} 1<\mathrm{T} * 255 * 0.95)$ Than fill Holes in Binary Image using Morphological Function Im Fill with Parameter 'Holes' after that Use Connected Component Labeling to Count Total number of Objects in Binary Image the Number of Blobs Found is RBC Count Estimate the it is stopped. [18]

Frist We Read Image of Stained Blood Cell Slide Than we Convert RGB Image to LAB Colour Space after that Reshape
Lab Images Number of Rows multiply By Number of Coloums 2 Than apply K-Means clustering on Reshaped Image Matrix Using Number of Cluster is 3 \& Obtain Cluster ID's after that Reshape Obtained Cluster ID's in Number of Rows Multiply by Number of Columns Than Re Map RGB Labels to Original RGB Image \& Obtain 3 Segmented Images after That take Compute Average Red, Green \& Blue Component of 3 Cluster Images than Compute Blue Content percentage of three Cluster Images after that Cluster OUT of Three Having Maximum Blue Percentage is Selected for Further Processing than Binarize Selected Cluster Image using Ostu's Method After that Fill Holes in The Binarized Image Than Remove Object Having Area Less than 300 Pixels after Thatlabel connected Components and Count Number of Objects the Number of Objects Correspond to Number of WBC's Than it has stopped. [19, 20]

\section{Image 1}

\section{Results}

We have taken this images and result for Blood cells stain image

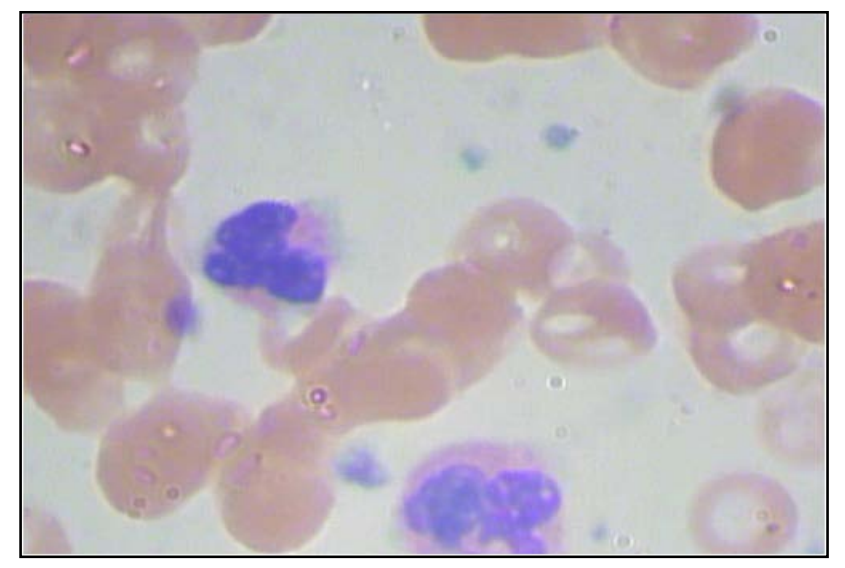

Fig.1:Input Image of Blood Cell Stain Image Tattled image 1

Table 1
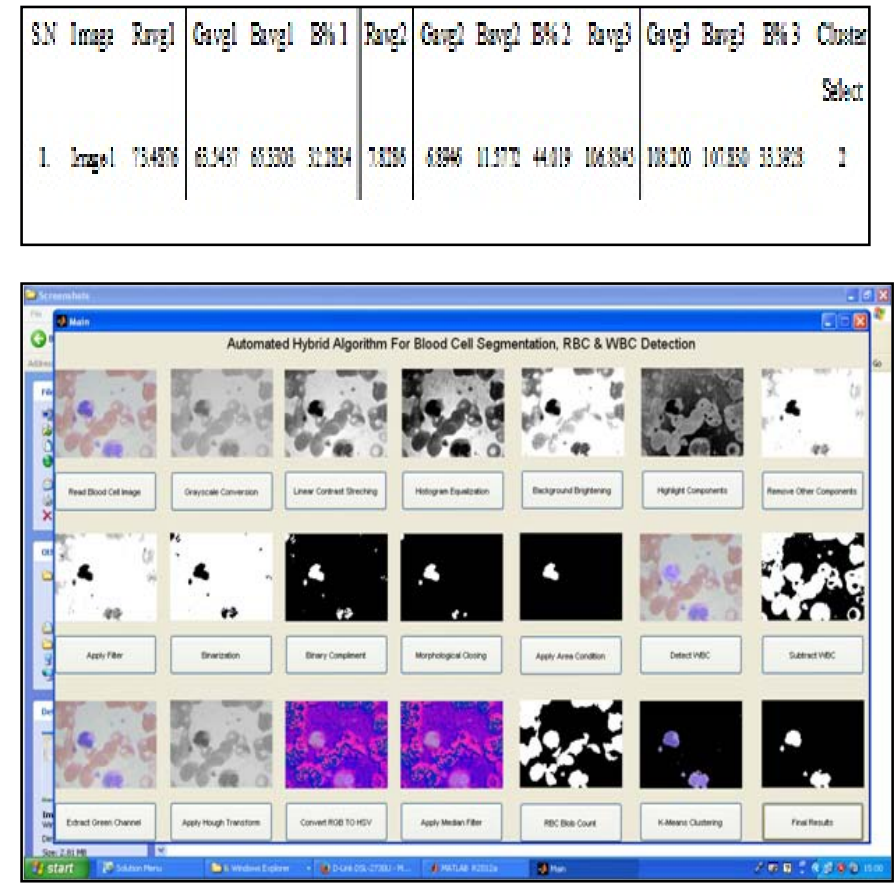
Fig.3: Automated Hybrid Algorithm for Blood Cell Segmentation RBC \&WBC Detection For Titteled Image 1

Table 2

\begin{tabular}{|c|c|c|c|c|c|c|c|c|c|}
\hline SN Inige RECLI & & RBCW & $B C W$ & BBCWA & WBCEN & IBCED. & BCWW. & WBC:CN & NBCENG \\
\hline $\begin{array}{c}1 . \log \\
1\end{array}$ & 9 & 3 & 4) & 1410 & 1 & 1 & 4 & $y$ & 200 \\
\hline
\end{tabular}

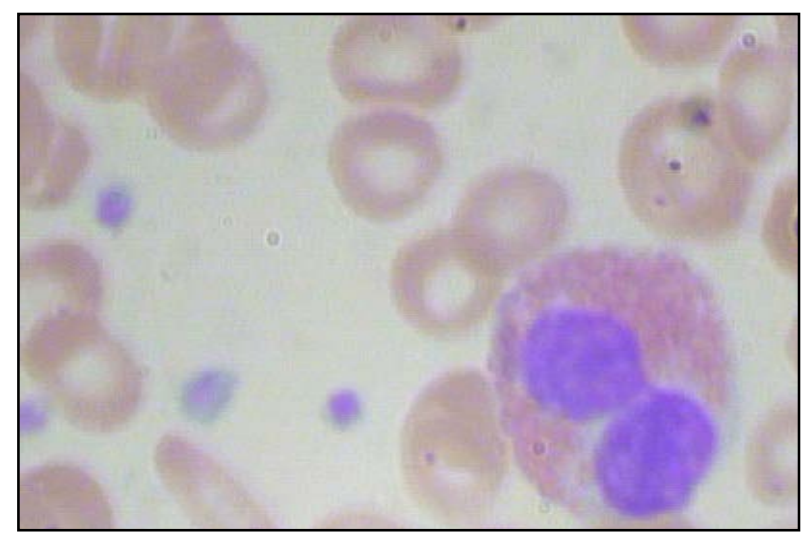

Fig.2:Input Image of Blood Cell Stain Image Tattled image 2

\section{Table 3}

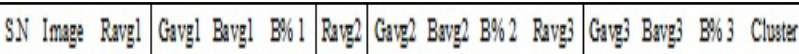

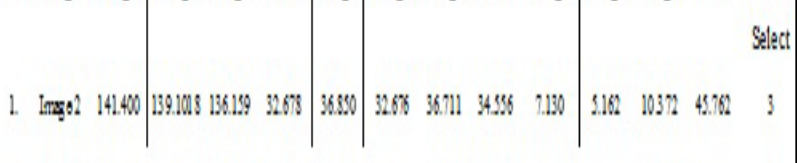

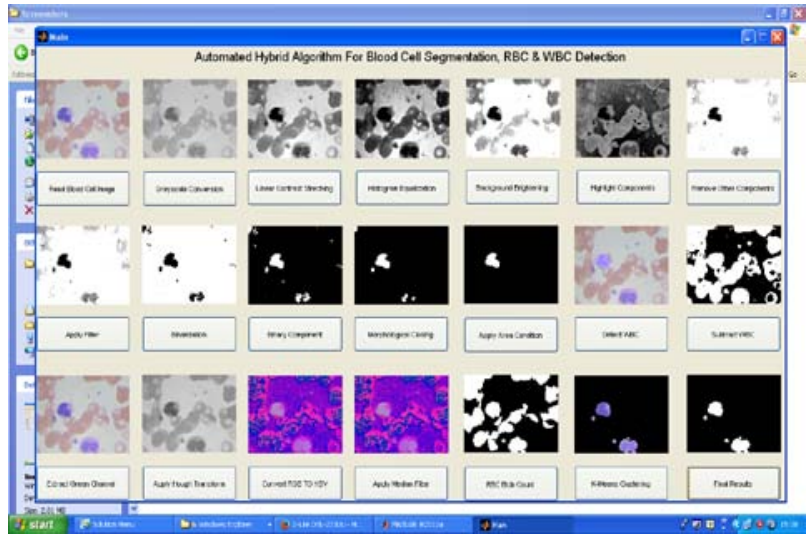

Fig.4:Automated Hybrid Algorithm for Blood Cell Segmentation RBC \&WBC Detection For Tattled Image 2

Table 4

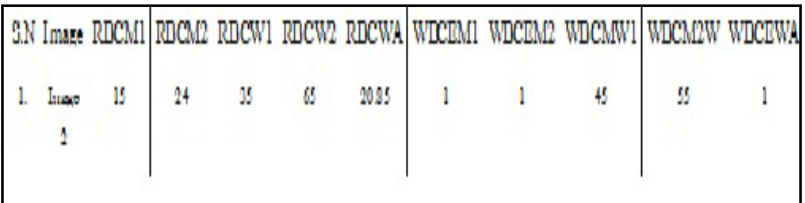

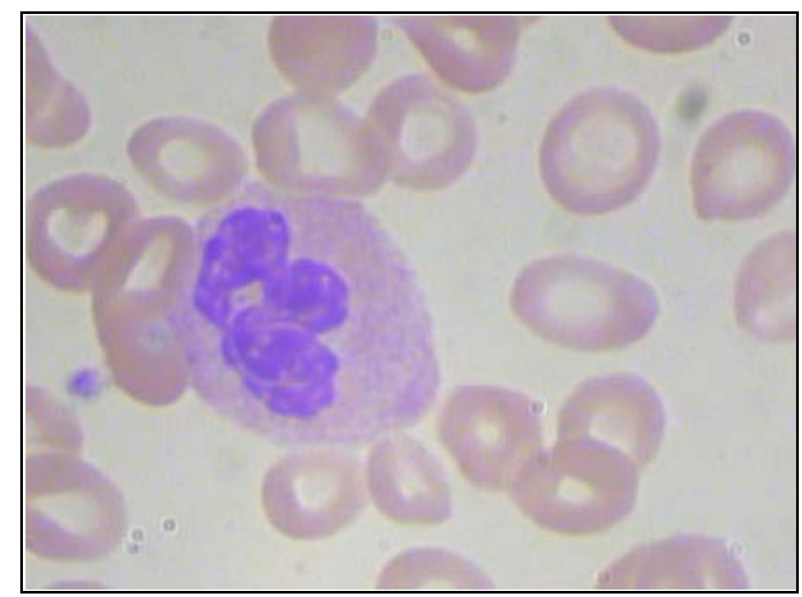

Fig.5:Input Image of Blood Cell Stain Image Tattled image 3

Table 5
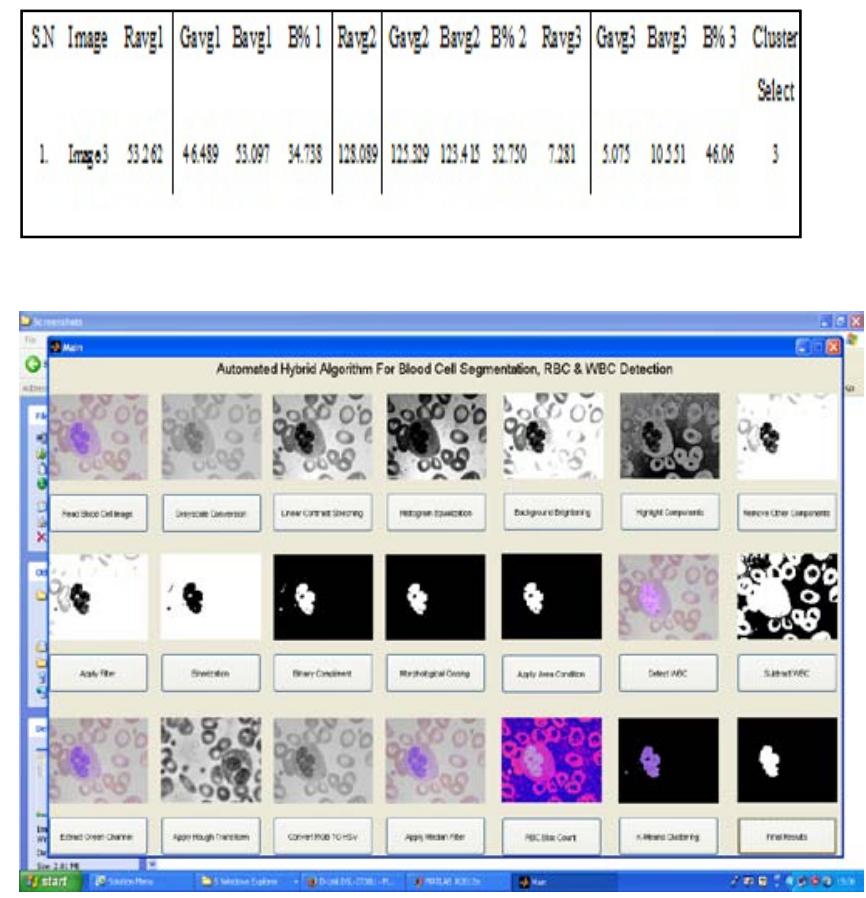

Fig.6: Automated Hybrid Algorithm for Blood Cell Segmentation RBC \&WBC Detection For Tattled Image 3

Table 6

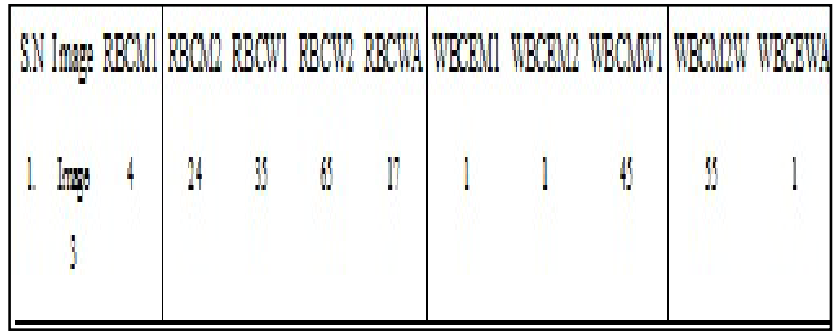


Table 9

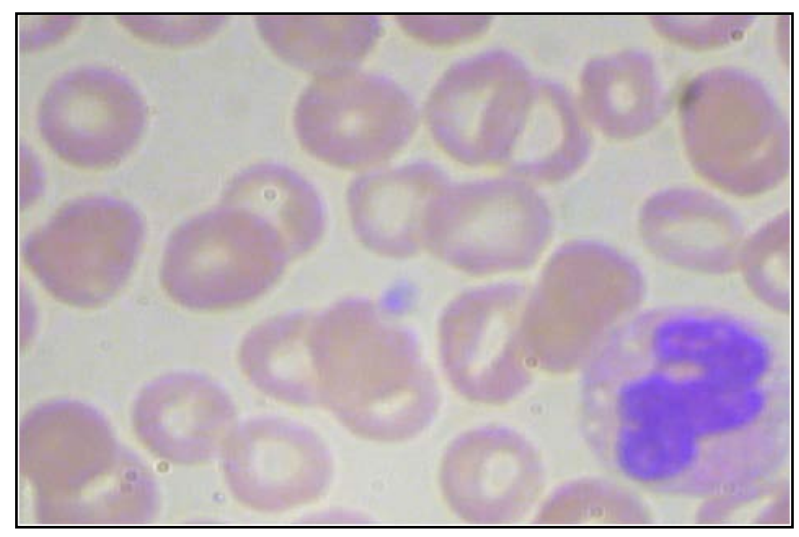

Fig.7 :Input Image of Blood Cell Stain Image Titteled image 4

Table 7

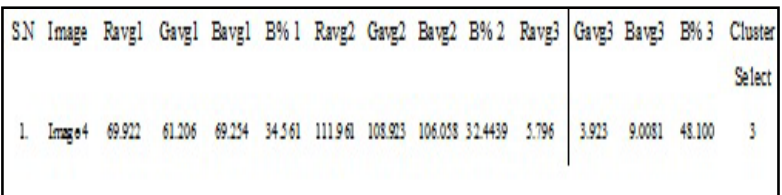

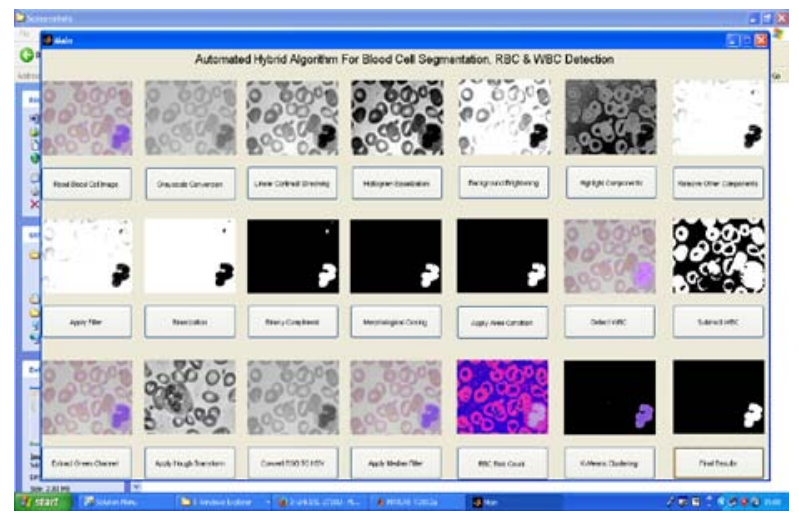

Fig.8: Automated Hybrid Algorithm for Blood Cell Segmentation RBC \&WBC Detection For Titteled Image 4

Table 8

\begin{tabular}{|c|c|c|c|c|c|c|c|}
\hline S.N Image RBCNII & RBCAN RBCW & $\mathrm{BCCW}$ & $\mathrm{RBCWA}$ & WBCE. & VBCEN & $\mathrm{BCCMW1}$ & WBCNEW WBCEWA \\
\hline 1. $\operatorname{lng}_{4} 23$ & 35 & 65 & mss & 1 & 1 & 45 & $\$$ \\
\hline
\end{tabular}

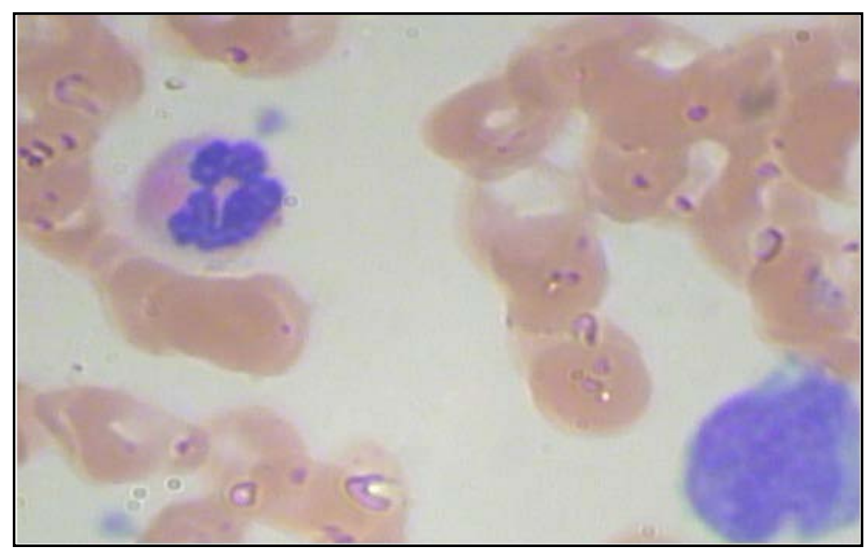

(C) 2015-19, IJARCS All Rights Reserved
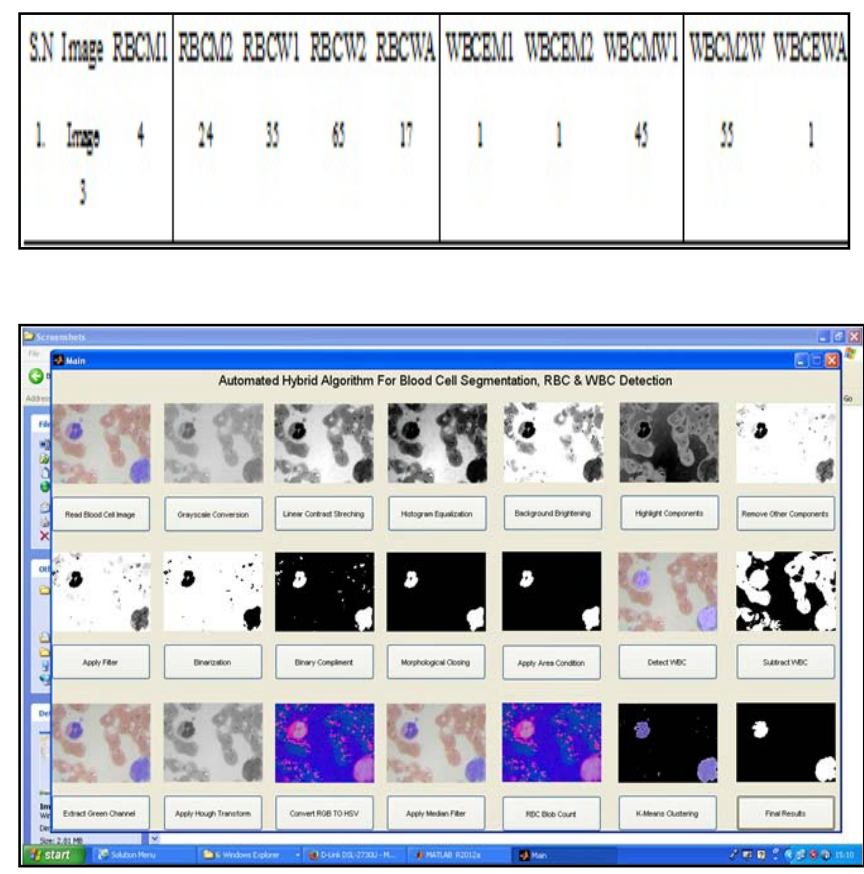

Fig.5.10: Automated Hybrid Algorithm for Blood Cell Segmentation RBC \&WBC Detection For Titteled Image 5

Table 10

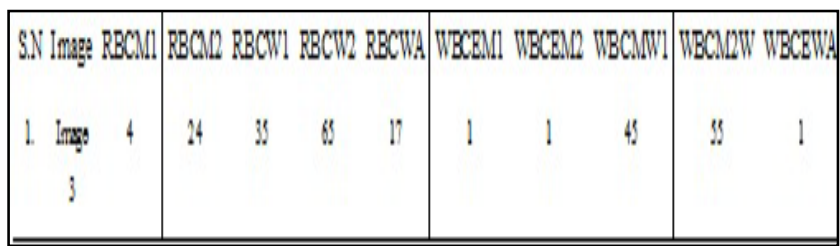

\section{Consolidate Table}

As

depicted in above table 11 , RGB avarage values for there Kmeans clusters have been consolidated for image 1 to image 5 . Also Bwc percentage of the clusters, and the selected WBC clbeen identified.

\section{Table 11}

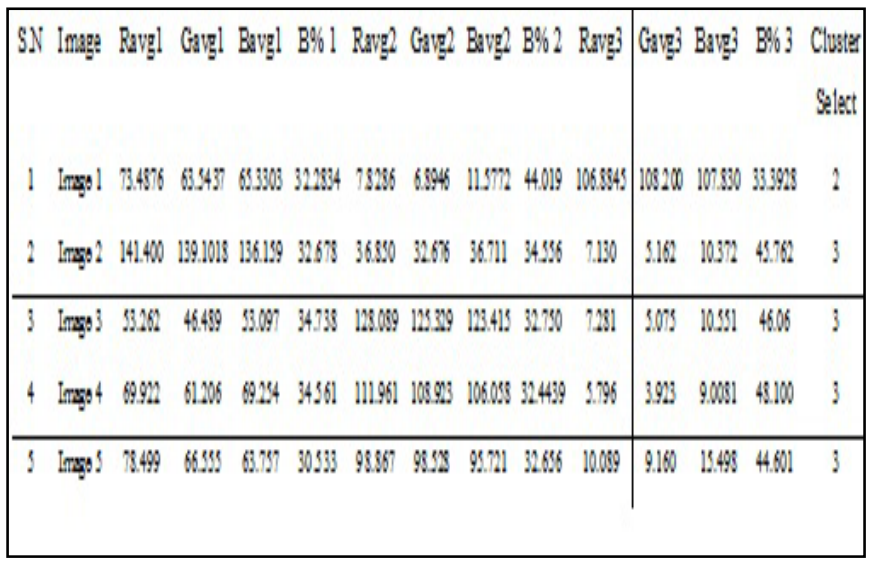

And table 12 is depicted RBC count estimate using method 1,RBC count method 2, their method weightages and their 
weighted average and WBC count estimate using method 1, WBC count estimate using method 2, their method weighted along with their weighted average is consolidated for image 1 to image 5 .

\section{Table 12}

\begin{tabular}{|c|c|c|c|c|c|c|c|c|c|c|}
\hline \multicolumn{2}{|c|}{ S.N Image RBCMI } & \multicolumn{4}{|c|}{ RBCW2 RBCW1 RBCW2 RBCWA } & \multicolumn{3}{|c|}{ WBCEM1 WBCEM2 WBCMW1 } & \multicolumn{2}{|c|}{ WBCMLW WBCEWA } \\
\hline $\begin{array}{c}1 . \operatorname{lng} \theta \\
1\end{array}$ & 19 & 9 & 38 & 68 & 11.100 & 1 & 1 & 45 & 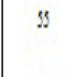 & 2680 \\
\hline $\begin{array}{c}2 \text { Imso } \\
2\end{array}$ & 15 & 24 & 38 & 68 & 2089 & 1 & 1 & 45 & $\$ 1$ & 1 \\
\hline $\begin{array}{c}3 \operatorname{limg} \theta \\
3\end{array}$ & 4 & 24 & 38 & 68 & 17 & 1 & 1 & 45 & 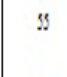 & 1 \\
\hline $\begin{array}{c}4 \operatorname{lngy} \\
4\end{array}$ & 33 & 30 & 38 & 68 & nss & 1 & 1 & 45 & 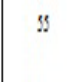 & 1 \\
\hline $1 \operatorname{lng} \theta$ & 15 & 11 & 38 & 68 & 12400 & 2 & 2 & 45 & 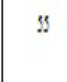 & 2 \\
\hline
\end{tabular}

Table 13

\begin{tabular}{|cccccccc|}
\hline S.N & Image Name & RBCWA & NBCWA & RBCNC & WBCMC & ?RBC & ? WBC \\
\hline 1. & Image 1 & 11.10 & 2.65 & 18 & 2 & 6.90 & -0.65 \\
2. & Image 2 & 20.85 & 1 & 18 & 2 & .2 .85 & 1 \\
\hline 3. & Image 3 & 17 & 1 & 14 & 3 & .3 .00 & 2 \\
4. & Image 4 & 27.55 & 1 & 22 & 1 & .5 .55 & 0 \\
5. & Image 5 & 12.40 & 2 & 11 & 1 & -1.40 & -1 \\
\hline & & & & & & $?=5.90$ & $?=1.35$ \\
\hline
\end{tabular}

In the table 13 depicted in above $\mathrm{RBC}$ weighted average method, WBC count weighted average method, RBC manual count, WBC manual count method, fiskBC value and $\triangle \mathrm{WBC}$ value than took sum $\triangle \mathbb{R B C}$ value and $\triangle \mathrm{WBC}$ value.

Than we find average value of $\triangle \mathrm{RBC}$

$$
\sum \Delta \mathrm{RBC} / 5=1.18
$$

and average value of $\triangle \mathrm{WBC}$

$$
\sum \Delta \mathrm{WBC} / 5=0.27
$$

\section{Conclusion}

In this work an innovative method has been proposed for a completely automatic analysis of blood cell parameters from microscopic blood slides. Thus our work provides for automatic blood parameter analysis support to medical creativity. The results offered show that the proposed method is able to count the number of RBC's and WBC's in a robust and reliable way. Also it is able to segregate WBC nucleus with command able accuracy. Weighted averaging system employed to assign weighted to the two defecation algorithms while averaging, for their improvement in the accuracy of the system. Thus the proposed system is a highly durable, low cost and time saving medical diagnostic setup. In this technique we founded $\triangle \mathrm{RBC}$ average value approximate 1.18 and $\triangle \mathrm{WBC}$ average value approximate 0.27 . These values are correct as with my point of view.

\section{FUTURE SCOPE}

As proposed the automation of blood analysis and parameters detection using image processing is a promising field. The work carried out in this dissertation adds value to previous work by improving accuracy of existing system, but a lot of more is required to be done to make this technology available for commercial usage. Improvement in accuracy further with artificial intelligence to detect image on mortality is mandatorily required for detection of various disease using RBC shape or WBC shape and nucleus parameterization can be included to provide for automated easily disease warning system using cutting edge nuclear technology, automated blood group detection can also be included in the proposed system, to mane the system a completely automatic human blood analysis system. For their advances in science and technologies in the forthcoming decode may provide for non invasive methods of blood parameter analysis is using portable (hunched) multiband Doppler.

\section{REFERENCES}

1. Qubilah D`souza, Sufola Das Chagas Silva Araujo, Dr. Luis C. Mesquita " Automated Eosinophil Cell count test of a Blood Semar as a Health Maker” IJESC 2017.

2. Bhagavathi S. L. and Thomas Niba S. "An Automatic system for Detectinh and Counting RBC \& WBC using Fuzzy Logic” ARPN 2016.

3. Kiran Tiwari , Prof. Mrs. Pooja Thakre "An Efficient Approach to Track RBC and Blood Diseases using Blood Sample” IJSTE 2016.

4. Megharani B. Chougale, Dr.T. B. Mohite-Patil "Automated Red Blood Cells Counting using Image Processing Techniques" IJSRD 2016.

5. Biji G, Dr.S.Hariharan "White Blood Cell Segmentation Techniques in Microscopic Images for Leukemia Detection" IOSR-JDMS 2016.

6. S. Raviraja*, S. D. Geethanjali, C. Chethana, B. M. Kanthesh "The Classification and Recognition of Plasmodium Parasite in Prediction of Malaria Infected Blood Smears Using Artificial Intelligence Technique” IJARCSSE 2015.

7. Awais Mansoor, PhD Ulas Bagci, PhD Brent Foster, BSc Ziyue $\mathrm{Xu}$, PhD Georgios Z. Papadakis, MD Les R. Folio, DO, MPH Jayaram K. Udupa, PhD Daniel J. Mollura, MD “Segmentation and Image Analysis of Abnormal Lungs at CT: Current Approaches, Challenges, and Future Trends” RSNA 2015.

8. S. Gowri, A. Kanagraj "Cancer tissue IdentificationUsing Monte Carlo Simulation and ICM Algorithm” IJMTES 2015.

9. Shailesh J. Mishra, Mrs A.P.Deshmukh "DETECTION OF LEUKEMIA USING MATLAB” IJARECE 2015.

10. Mr. Mehdi Habibzadeh Motlagh "Automatic Segmentation and Classification of Red and White Blood cells in Thin Blood Smear Slides” MHM 2015.

11. M.MumthajBegam, R. Geetha and A. Sagayaselvaraj Red Blood Cell Identification Using Watershed Technique IJRASET 2015.

12. K shipra Charpe, V.K. Bairagi, Shama Desarda, Sheetal Barshikar "A Novel Method for Automatic Detection of Malaria Parasite Stage in Microscopic Blood Image” IJCA 2015.

13. Mehedi Hasan Talukder, Md. Mahfuz Reza , Mahbuba Begum, Md. Rabiul Islam , Md. Mahmudul Hasan "Improvement of Accuracy of Human Blood Groups Determination using Image 
processing Techniques” IJARCCE 2015.

14. Mrs. Sonali, C. Sonar, Prof. K. S. Bhagat "An Efficient Technique for White Blood Cells Nuclei Automatic Segmentation” IJSER 2015.

15. Malhar Bhatt, Shashi Prabha "Detection Of Abnormal Blood Cells Using Image Processing Technique” IJEEE 2015

16. Senthilbabu D, Maheswari "White Blood Cell Segmentation using hybrid segmentation methods” IJERT 2014.

17. Priyadharshini.R, Ramya.S, Kalaiyarasi.S, Kalpana Devi.S, SuthathiraVanitha.N "A Novel Approach In Identification Of
Blood Group Using Laser Technology” IJERT 2014.

18. Nur Alom Talukdar, Daizy Deb Sudipta Roy “Automated Blood Cancer Detection Using Image Processing Based on Fuzzy System” IJARCSSE 2014.

19. Hariganesh S, Vanitha.K "Review on Blood Platelet Transfusion Using Data Mining” IJESI 2014.

20. Tejaswini H V, M S Mallikarjuna Swamy "Determination and Classification of Blood Types using Image Processing Techniques” ITSI-TEEE 2014. 